\title{
Multi-wavelength study of Galactic star-forming regions with near-infrared instruments on $2-4$ meter class Indian telescopes
}

\author{
DEVENDRA K. OJHA
}

\begin{abstract}
We present a brief description of the activities of the infrared astronomy group of Tata Institute of Fundamental Research with special emphasis on the near-infrared instrumentation for star formation studies using 2 - 4-meter class Indian ground-based telescopes.
\end{abstract}

Key words: ISM, star formation, infrared, NIR instrumentation.

\section{INTRODUCTION}

The central research theme of the infrared astronomy (IRA) group at Tata Institute of Fundamental Research (TIFR) is the study of interstellar medium (ISM) in relation to star formation in our Galaxy and nearby galaxies. The study of ISM provides a powerful probe into the physical and chemical properties of the interstellar dust and gas, which predominantly emits in the infrared waveband. Infrared emitting ISM is a tracer of several important astrophysical phenomena, namely, star formation activity, shock front, material recycling, photo processes in the proximity of young stars, and plasma cooling. The corresponding research activities are currently executed using TIFR's own ground-based near infrared (NIR) imagers \& spectrometers, TIFR's indigenously developed $100-\mathrm{cm}$ balloon borne far-infrared (FIR) telescope, national optical imagers \& spectrometers, the Giant Metrewave Radio Telescope as well as international facilities such as the large aperture ground-based telescopes and astronomical satellites. The observations are complemented with interpretation backed by image processing as well as numerical modelling (e.g., radiative transfer) schemes based on codes developed in-house as well as those available publicly. In addition to the above activities, the group actively participates in instrument development for ground-based and space-based astronomy.

With the above aim, the IRA group designed and built the TIFR Near Infrared Spectrometer and Imager (TIRSPEC) in collaboration with M/s Mauna Kea Infrared, LLC, Hawaii (hereafter MKIR) during the 2007-2012 five-year period, now in operation on the side port of the 2-meter Himalayan Chandra Telescope (HCT), Hanle (Ladakh), India, at an altitude of 4550 meters above mean sea level (see details in Ojha et al. 2012a, Ninan et al. 2014). Recently, the IRA group has also upgraded the TIFR Near Infrared Imaging Camera-II (TIRCAM2) (Ojha et al. 2012b, Naik et al. 2012) which was 
being used with the 2-meter Inter-University Centre for Astronomy and Astrophysics (IUCAA)'s Girawali observatory telescope, near Pune. This instrument has been tested by the IRA group at the 3.6-meter Devasthal Optical Telescope (DOT) and is being used by Indian astronomers since May 2017 onwards. Besides this, TIFR has also been observing in the FIR band (120 to $220 \mu \mathrm{m}$ ) using the 100-cm balloon borne FIR telescope (Ghosh et al. 1988, Ghosh 2010). Recently, as a part of the TIFR-Japan collaboration in balloon-borne FIR astronomy, the TIFR $100-\mathrm{cm}$ balloon-borne FIR telescope along with the Japanese Fabry-Perot Spectrometer, tuned to the astrophysically interesting [C II] fine structure line at $157.74 \mu \mathrm{m}$, has been successfully flown several times to map large regions in [C II] line and continuum of several northern and southern star-forming complexes (Mookerjea et al. 2001, 2003, Kaneda et al. 2013). To complement these studies in the NIR band, the need was felt for a dedicated Optical-NIR spectrometer in the 0.5 to $2.5 \mu \mathrm{m}$ range, which could be used with the 3.6-meter DOT. To meet this need, the TIFR-ARIES Near Infrared Spectrometer (TANSPEC) (Ojha et al. 2012b, Ojha et al. 2018a,b) was conceived to provide spectroscopy in the range from 0.55 to $2.54 \mu \mathrm{m}$, with a spectral resolving power of $\sim 2750$ to be used on the axial port of the 3.6-meter DOT.

In this paper, we describe the technical details of TIRCAM2, TIRSPEC and TANSPEC for star fomation studies in India.

\section{TIFR NEAR INFRARED IMAGING CAMERA-II (TIRCAM2)}

The TIFR Near Infrared Imaging Camera-I (TIRCAM1) having a $58 \times 62$ pixels InSb focal plane array (FPA), was the first array camera developed by our group. Using the TIRCAM1, observations were mainly carried out during the period 2001-2006 at the focal plane of the 1.2-meter Mount Abu infrared telescope ( $f / 13$ Cassegrain focus) of Physical Research Laboratory, Ahmedabad. Later in 2012, the camera was upgraded with a larger Raytheon $512 \times 512$ pixels InSb Aladdin III Quadrant FPA. The optics was re-designed for use of the camera at the focal plane of the 2-meter HCT (f/g) operated by Indian Institute of Astrophysics, Bangalore, and the 2-meter Girawali Telescope $(f / 10)$ operated by IUCAA, Pune. The camera was therefore renamed as TIRCAM2. The detector is cooled to an operating temperature of $35 \mathrm{~K}$ and the optical elements to $17 \mathrm{~K}$ by a closed-cycle Helium cryo-cooler. The InSb array in the TIRCAM2 is sensitive to photons in the 1-5 $\mu \mathrm{m}$ wavelength band. However, the optics in the TIRCAM2 restricts the operating wavelength range below $\sim 3.8 \mu \mathrm{m}$. The camera accommodates seven selectable standard NIR filters for imaging observations.

The TIRCAM2 was recently mounted at the axial port of the 3.6-meter DOT, the largest optical aperture in the country till date. The camera offers a field-of-view (FoV) of $\sim 86^{\prime \prime} .5$ $x 86^{\prime \prime} .5$ on the DOT with a pixel scale of $\mathrm{O}^{\prime \prime} .169$. The performance of the TIRCAM2 on the 3.6-meter DOT is found to be consistent with the expectations. At longer wavelengths, specifically at the $n b L$-band $\left(\lambda_{\text {cen }} \sim 3.59 \mu \mathrm{m}\right)$, the results are highly encouraging. In spite of high humidity (>70\%) during the calibration observation runs, the seeing was generally sub-arcsecond, and the best seeing obtained was $\sim 0.45$ arcsec in the $K$-band on October 16, 2017. Deep imaging observations show that the camera has the capability to observe sources up to $19.0 \mathrm{mag}$, $18.8 \mathrm{mag}$, and 18.0 mag with 10\% photometric accuracy in $J, H$ and $K$ bands, respectively. The camera is also capable of detecting the $n b L$-band sources brighter than $\sim 9.2 \mathrm{mag}$, and hence, can be useful in observing bright sources that are saturated in the Spitzer-IRAC $3.6 \mu \mathrm{m}$ 
and the WISE W1-band images. Overall, it is found that the TIRCAM2 with the 3.6-meter DOT is adequate for deep NIR observations that are comparable to other 4 -meter class telescopes available world-wide. The camera is also capable of detecting strong Polycyclic Aromatic Hydrocarbons (PAH) emitting sources $\left(F_{3.3 \mu m} \geq 0.4 \mathrm{Jy}\right.$ ), like Sh 2-61 (see details in Ojha et al. 2018a,b; Baug et al. 2018).

The TIRCAM2 is proposed to be used on one of the side ports of the DOT for regular science observations as the axial port will be occupied by one of the other larger instruments (e.g., TANSPEC). It was successfully tested on one of the side ports of the largest Indian telescope, DOT, in collaboration with ARIES, Nainital. The first light from this port was seen on May 4 , 2020. This achievement is significant since the efficiency of the telescope will drastically increase with two instruments being available for near simultaneous observations during any particular night.

It is noteworthy to mention that TIRCAM2 is the only instrument of its kind in India which is sensitive up to $3.8 \mu \mathrm{m}$ and is ideal for the imaging of astrophysical sources at wavelengths longer than $2 \mu \mathrm{m}$. One of the scientific aims of TIRCAM2 is to image the Galactic star-forming regions in the $3.3 \mu \mathrm{m}$ PAH band using intelligently planned narrow band filters.

\section{TIFR NEAR INFRARED SPECTROMETER AND IMAGER CAMERA (TIRSPEC)}

We have designed and built a near-infrared spectrometer and imager (TIRSPEC) in collaboration with MKIR. The TIRSPEC is based on $1024 \times 1024 \mathrm{HgCdTe}$ focal plane array (Rockwell HAWAII-I 1K PACE detector), for 2-meter HCT. The development process of the TIRSPEC was completed in November 2012 and it was assembled, tested and shipped to TIFR in
December 2012. The TIRSPEC was commissioned and the laboratory tests were conducted in TIFR to qualify and calibrate the performance of the spectrometer in the lab. At the f/9.2 focus of the 2-meter HCT, TIRSPEC image scale is $0^{\prime \prime} .3$ per pixel $(18.5 \mu \mathrm{m})$, covering a $307^{\prime \prime} \times 307^{\prime \prime}$ FoV. The camera has the capability to observe sources up to $18.0 \mathrm{mag}, 17.5 \mathrm{mag}$, and $17.0 \mathrm{mag}$ with $10 \%$ photometric accuracy in $J, H$ and $K$ bands, respectively. TIRSPEC is equipped with 3 grisms. One is the YJHK grism that allows single order spectroscopy in the $J, H$ and $K$ windows. The $Y J$ and $H K$ cross dispersing grisms allow simultaneous $Y \& J$ and $H$ \& $K$ spectroscopy in the cross-dispersed mode, respectively. The TIRSPEC is also equipped with a number of broad- and narrow-band filters. The wide-band filter set includes $J, H$ and $K_{\mathrm{s}}$ standard filters. The narrow-band filter set includes $\mathrm{H}_{2}$ 1-O S(1), $\mathrm{Br}$ Gamma, $\mathrm{CO}$ 2-O, [Fe II] and $\mathrm{CH}_{4}$ filters, plus the associated $K$ and $H$ narrow-band filters to sample the near-by continuum.

After the completion of all laboratory tests in the lab, the TIRSPEC was shipped and integrated on the side port of the HCT at Hanle (Ladakh) in June 2013 for first light observations. It has undergone engineering and science runs on the telescope during June - September 2013. The regular science observations with the TIRSPEC started from October 2013. The TIRSPEC was successfully characterized and thrown open to the global astronomy community from May 1, 2014. Subsequently, subarray readout capability has been incorporated into the TIRSPEC system to allow for photometry of brighter objects. Currently, about $50 \%$ of the observing proposals on 2-meter HCT use TIRSPEC as the focal plane instrument. Recently, long duration exposure for faint source spectroscopy has been introduced in TIRSPEC and initial analysis shows this seems to be giving better $\mathrm{S} / \mathrm{N}$ ratio. 


\section{TIFR-ARIES NEAR INFRARED SPECTROMETER (TANSPEC)}

We have developed a near-infrared spectrograph (TANSPEC), as a collaboration project between TIFR, ARIES, Nainital, and MKIR, to be used on the main port of the 3.6-meter DOT. The TANSPEC Preliminary Design Review (PDR) involving national and international experts was carried out during July 20 to 24,2015 at ARIES, Nainital. After the successful completion of the PDR, the Critical Design Review of TANSPEC was held during October 2015. The spectrograph operates in two modes whereby the spectrum is focussed on to a $2 \mathrm{k} \times 2 \mathrm{k} \mathrm{H} 2 \mathrm{RG}$ array. In the cross-dispersed mode, a combination of a grating and two prisms are used to pack all the orders on to the $\mathrm{H}_{2} \mathrm{RG}$ array at a resolution of $\mathrm{R} \sim 2750$ in the $\mathrm{K}$-band. A range of slit widths are available from 0.5 arcsec to 4.0 arcsec. It also has a low resolution prism mode $(R \sim 100$ - 350) for high throughput observations. The instrument also has an independent imaging camera with a $1 \mathrm{k} \times 1 \mathrm{k} \mathrm{H} 1 \mathrm{RG}$ detector which acts as the infrared guider and also NIR imager. The reflected beam from the mirrored slit is imaged to this camera through a filter wheel which consists of broad band $r^{\prime}, i^{\prime}, Y, J, H$ and $K_{\mathrm{s}}$ and narrow band $\mathrm{H}_{2}$ \& $\mathrm{Br} \gamma$ filters. This camera has a field of view of 1 arcmin $\times 1$ arcmin, and is used for guiding the telescope (IR guider) as well as imaging for photometry. It also functions as a pupil viewer for instrument alignment on the telescope. The first cool down tests of TANSPEC (after installation of optics and detectors) were successfully completed in December 2017. The TANSPEC was shipped to DOT on March 10, 2019. It has undergone engineering and commissioning runs on the telescope during April - May 2019 and December 2019.

The first light of this instrument on the DOT was observed on April 13, 2019. The TANSPEC is currently being commissioned on the DOT and will be available for science observations from October 2020. The TANSPEC was also thrown open to ARIES and TIFR users during February 2020 to get hands-on training and science utilisation. The performance of the TANSPEC on the 3.6-meter DOT is found to be consistent with the expectations. The TANSPEC will be a unique spectrograph which will provide simultaneous wavelength coverage from $0.55 \mu \mathrm{m}$ (optical) to $2.54 \mu \mathrm{m}$ (NIR) with a resolving power of $\mathrm{R} \sim 2750$ at $2.2 \mu \mathrm{m}$.

\section{CONCLUSIONS}

TIRSPEC, TANSPEC and TIRCAM2 at the focal planes of the 2-meter HCT and 3.6-meter DOT will be a major workhorse for a variety of challenging astrophysical problems. These will be extremely sensitive to low temperature stellar photospheres $(T \leq 2500 \mathrm{~K})$ and objects surrounded by warm dust envelopes or embedded in dust/molecular clouds. These NIR instruments are therefore particularly suited to the search for low and very low mass stellar populations (M dwarfs, brown dwarfs), strong mass-losing stars on the asymptotic giant branch, young stellar objects still in their protostellar envelopes and active galactic nuclei.

The preliminary results from the earlier observing runs of TIRCAM2 with the DOT were encouraging, particularly at longer wavelengths $(>2 \mu \mathrm{m})$. This will further allow us to explore the capability of the TIRCAM2 at longer wavelength, particularly in the L-band. The TIRCAM2 is also proposed to be used on one of the side ports of the DOT since the axial port will be occupied by other main ARIES instruments (e.g., TANSPEC) in the near future. TANSPEC will be a unique spectrograph which will provide simultaneous wavelength coverage from $0.55 \mu \mathrm{m}$ (Optical) to $2.54 \mu \mathrm{m}$ (NIR) with a resolving power of $\mathrm{R} \sim 2750$. 


\section{Acknowledgments}

We thank the anonymous reviewers for useful comments and suggestions. We also thank the staff at the 3.6-meter DOT, Devasthal (ARIES), for their co-operation during TIRCAM2 and TANSPEC observations. It is pleasure to thank the members of the IR astronomy group at TIFR for their support during observations. We thank the staff of IAO, Hanle and CREST, Hosakote, that made TIRSPEC observations possible. We would also like to thank Mr. Douglas W. Toomey and the entire MKIR team for their contribution in the TIRSPEC and TANSPEC projects. DKO acknowledges the support of the Department of Atomic Energy, Government of India, under project No. 12-R\&D-0200.TFR-5.02-0200.

\section{REFERENCES}

BAUG T ET AL. 2018. TIFR Near Infrared Imaging Camera-II on the $3.6 \mathrm{~m}$ Devasthal Optical Telescope. J Astron Instrum 7: 1850003-11.

GHOSH SK. 2010. Infrared astronomy in India with balloons. Interstellar Matter and Star Formation: A Multi-wavelength Perspective. ASI Conference Series 1: 167-170.

GHOSH SK, IYENGAR KVK, RENGARAJAN TN, TANDON SN, VERMA RP \& DANIEL RR. 1988. Far-infrared (120-300 micron) observations of the Carina nebula. Astrophys J 330: 928-936.

KANEDA H, NAKAGAWA T, GHOSH SK, ISHIHARA D, KONDO T, NINAN JP, TANABE M, FUKUI Y, HATTORI Y, ONAKA T, TORRI K \& YAMAGISHI M. 2013. Astronomy \& Astrophysics 556: A92, 1-9.

MOOKERJEA B, GHOSH SK, KANEDA H, NAKAGAWA T, OJHA DK, RENGARAJAN TN, SHIBAI H \& VERMA RP. 2001. Mapping of the Orion molecular cloud in the [CII] line and continuum at $158 \mu \mathrm{m}$. B Astron Soc India 29: 337-338.

MOOKERJEA B, GHOSH SK, KANEDA H, NAKAGAWA T, OJHA DK, RENGARAJAN TN, SHIBAI H \& VERMA RP. 2003. Mapping of large scale $158 \mu \mathrm{m}$ [CII] line emission: Orion A. Astronomy \& Astrophysics 404: 569-578.

NAIK MB ET AL. 2012. TIRCAM2: The TIFR near infrared imaging camera. B Astron Soc India 40: 531-545.

NINAN JP ET AL. 2014. TIRSPEC: TIFR Near Infrared Spectrometer and Imager. J Astron Instrum 3: 1450006-15.

OJHA DK, GHOSH SK, D'COSTA SLA, NAIK MB, SANDIMANI PR, POOJARY SS, BHAGAT SB, JADHAV RB, MESHRAM GS \& BAKALKAR CB. 2012a. TIFR Near Infrared Spectrometer and Imager (TIRSPEC). Recent Advances in Star Formation: Observations and Theory. ASI Conference Series 4: 191-192.

OJHA DK ET AL. 2012b. First light observations with TIFR Near Infrared Imaging Camera (TIRCAM-II). Recent Advances in Star
Formation: Observations and Theory. ASI Conference Series 4: 189-190.

OJHA DK ET AL. 2018a. Prospects of star formation studies with near-infrared instruments on 2-4 meter class Indian ground-based telescopes. The Cosmic Wheel and the Legacy of the AKARI archive: from galaxies and stars to planets and life: 257-260

OJHA DK ET AL. 2018b. Prospects for star formation studies with infrared instruments (TIRCAM2 and TANSPEC) on the 3.6-m Devasthal Optical Telescope. Bulletin de la Société Royale des Sciences de Liège, in Proceedings of the First Belgo-Indian Network for Astronomy \& Astrophysics (BINA) workshop 87: 58-67.

\section{How to cite}

OJHA D. 2021. Multi-wavelength study of Galactic star-forming regions with near-infrared instruments on $2-4$ meter class Indian telescopes. An Acad Bras Cienc 93: e2O200838. DOI 10.1590/0001-3765202020200838.

\section{DEVENDRA K. OJHA}

https://orcid.org/0000-0001-9312-3816

Department of Astronomy and Astrophysics, Tata Institute of Fundamental Research, Homi Bhabha Road, Colaba, Mumbai 400005 , India

E-mail:ojha@tifr.res.in

Manuscript received on May 31; accepted for publication on July 8,2020

\section{(cc) BY}

